\title{
GI-MS48-P09 | CRENEL OR NOT CRENEL, WHAT IS THE FUnCTION?
}

Duverger-Nédellec, Elen (Charles University, Faculty of Math. \& Phys., DCMP, Praha 2, CZE); Bretosh, Kateryna (Laboratoire de Chimie de Coordination du CNRS, Toulouse, FRA); Béreau, Virginie (Laboratoire de Chimie de Coordination du CNRS, Toulouse, FRA); Duhayon, Carine (Laboratoire de Chimie de Coordination du CNRS, Toulouse, FRA); Sutter, Jean-Pascal (Laboratoire de Chimie de Coordination du CNRS, Toulouse, FRA); Petrícek, Václav (Institute of Physics CAS, Department of Structure Analysis, Prague 8, CZE)

Modulated structures are not a piece of cake to solve but some of them can be trickier than the others. The most obvious solution is not always the proper one and conscientious verifications of the model have to be done in order to obtain the best structural model. We will focus here on the example of the $(3+1) D$ structure of the FeLN5PhenMeCl2 complex presenting a typical misleading modulation. The resolution and refinement of the structure were performed using the software Jana2006 [1]. The average structure of FeLN5PhenMeCl2 was determined using Superflip program. The structure is composed of two superimposed inversed configurations of the complex, indicating the presence of disorder. This disorder is the tricky point of the structural resolution! To model it, two options can be considered: the use of a crenel-type occupational modulation or the use of a sinusoidal occupation-function coupled with a positional modulation function. In the first case, the disorder observed in the average structure is, in fact, a hidden-order: both configurations would exist independently, appearing alternately along the periodicity axis of the modulation. In the second case, the disorder can reflect an ordered-disorder along the fourth dimension: each configuration presents a positional modulation (the order) and a probability of presence given by the occupational modulation (the disorder). Both possibilities will be investigated. Final structures will be observed via the new graphic tool developed in Jana2020 enabling the direct observation of mixed modulations on the structure.

[1] V. Petříček et al (2014), Z. Kristallogr. 229(5), 345-352. 